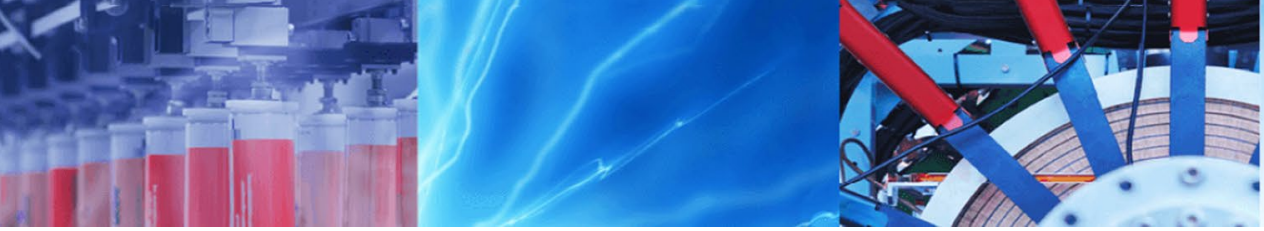

Research Article

\title{
Development and evaluation of drug delivery patch for topical wound healing application
}

\author{
Sadia Hassan ${ }^{1} \cdot$ Murtaza Najabat Ali $^{1}$ (D) Mariam Mir ${ }^{1} \cdot$ Ammad Ahmed $^{1} \cdot$ Munam Arshad $^{1}$
}

Received: 1 April 2021 / Accepted: 15 September 2021

Published online: 29 September 2021

(c) The Author(s) 2021 OPEN

\begin{abstract}
Wound treatment remains a challenge to many clinicians because of the complexities of the wound healing process. With the astonishing progress of biomedical engineering during the past few decades, conventional drug delivery systems have been evolved into smart drug delivery systems with stimuli-responsive characteristics. The objective of this study was to develop and evaluate an electromechanically actuated drug dispensation device which can release active pharmaceutical compound in a controlled fashion. Additive manufacturing was employed to design and fabricate the device. Haptic technology was used to provide stimulation for drug release, and Cicatrin was used to evaluate the drug release patterns of device. Drug release study was comprised of in vitro drug release, static study, and the purpose of this study was to develop a compliance chart for different wound conditions. The effectiveness of shortlisted drug regimen from compliance chart was validated through microbial study and animal studies. The results of animal studies were compared with commercially available drug release systems. The results of drug release studies gave different dose regimens for different wound conditions. The effective dose regimen was able to create $1-\mathrm{cm}$-wide microbial zone of inhibitions. The wound healing rate of mice for commercially available release system for five consecutive days was $10 \%, 10 \%, 20 \%, 40 \%$ and $50 \%$ and for test device was $10 \%, 30 \%, 60 \%, 90 \%$ and $100 \%$. Hence, the device proved its effectiveness and efficacy of dosage regimen for wound healing applications through in vitro, microbial and in vivo studies. In conclusion, this device proved to be an accurate and specific drug delivery system with improved medication and therapeutic outcomes for personalized medication.
\end{abstract}

Keywords Smart bandage · Drug delivery · Topical wounds · Chronic wounds · 3D printing

\section{Introduction}

An injury to the body by any means which is responsible for disruption and loss of continuity of the epithelial layer of skin and damages the underlying layers is termed as wound [1]. Wound heals through a complex and dynamic process of healing which involves many players at cellular and molecular level, primarily cytokines, growth factors and regulatory moieties. For a healthy patient, interleukin IL-1 is released to signal the damage and through homeostasis, body prepares the damaged site to clot the blood and to undergo inflammation which attracts the nutrients, enzymes, leukocytes, macrophages and monocytes to the affected area resulting in excessive exuded production. After the successful inflammation, the new matrix is formed by the aid of granulation tissues, collagen and fibroblasts, in the presence of sufficient oxygen, blood and nutrients [2]. Because of absence of standard conditions, i.e., oxygen, blood, nutrients, growth factors and sensory response, and presence of infectious agents, irregular

Murtaza Najabat Ali, murtaza.bme@hotmail.com | ${ }^{1}$ Department of Biomedical Engineering \& Sciences, School of Mechanical and Manufacturing Engineering, National University of Sciences and Technology (NUST), Sector H-12, Islamabad, Pakistan.

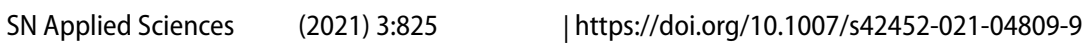


metabolites and immuno-suppressants, the wound heals without restoring the anatomical and physiological functions. These wounds are unable to pass through inflammatory check-post which prolongs the inflammatory response resulting in jogging in a vicious cycle between inflammation and angiogenesis [3]. The delayed wound healing seems a simple issue but when it posed a burden of 28.1 billion USD in 2019 on economy of single country, it became a major issue of modern world [4].

There are many reasons of transition of simple wound into a chronic wound, i.e., diabetes, burns and other metabolic issues but major reason lies in administration of wounds as the commercially available administration tools (gels, ointments, liquids) are passive drug release systems and their drug release is characterized as uncontrolled and immediate. Because these systems release an uncontrolled amount of drug, there is a possibility of releasing of drug dosage higher than the toxic level or lesser than the effective therapeutic level [5]. The maintenance of drug dosage at effective therapeutic level is very important for efficient wound healing and passing each phase accurately. The maintenance of effective therapeutic level can be achieved when the drug dosage is controlled according to the wound conditions (neither more than toxic level nor less than effective therapeutic level) and when patient or clinicians exactly know how much drug is being delivered at the wound site. Scientists have introduced controlled released systems for that purpose, and some are commercially available like Insulin Pen and Synchromate B. Unfortunately, there is no such device for wound healing purpose.

Micro-reservoir systems have been reported since 1987 where the researchers used segmented polyurethane for as drug reservoirs [6]. There are different types of reservoir systems but all of them are for liquid drugs. The current system is developed using micro-reservoir concept, but it is merged with electronics and it is specifically for powdered drug. In this device, vibrations are combined with electronics to deliver the pulsating dose within seconds when required. The device is prepared in a prepackaged form which will contain the drug for different wound sizes. This drug delivery mechanism was applied with a commercially available antibiotic Cicatrin to analyze the drug delivery behavior, to see the effectiveness of dosage against wound pathogens and to compare with commercially available passive drug delivery systems in animal models.

\section{Materials and methodology}

\subsection{Materials}

The initial investigation was done on acrylonitrile butadiene styrene (ABS) which was provided by Smooth-On Inc. PA, USA and linear resonant actuator was provided by Precision Microdrives. ABS (Polyacrylonitrile-ButadieneStyrene) 3D printing filament, nutrient agar, cicatrin powder and phosphate buffered saline tablets were provided by Sigma-Aldrich.

\subsection{Design idea}

The smart bandage consists of three components. First component is ABS-based plastic platform, vibration system and pharmaceutically active ingredient. The plastic platform has three enclosed compartments, two of which act as drug reservoirs and one houses the vibratory device. The vibratory device is battery-operated Linear Resonate Actuator (LRA). This device is controlled by microcontroller [7]. The LRA delivers the vibration stimulus to the drug reservoirs upon pressing the switch button. The drug reservoirs are controlled by microcontroller through LRA, and it controls the amount of drug delivery according to our desired levels. Microcontroller is be attached to switch button so that the patient can deliver drug at desired intervals Fig. 1.

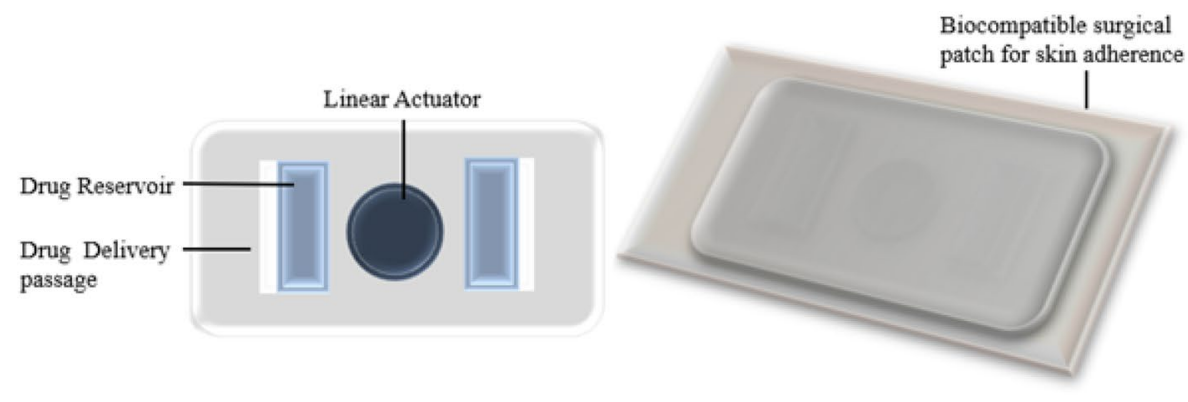

(a)

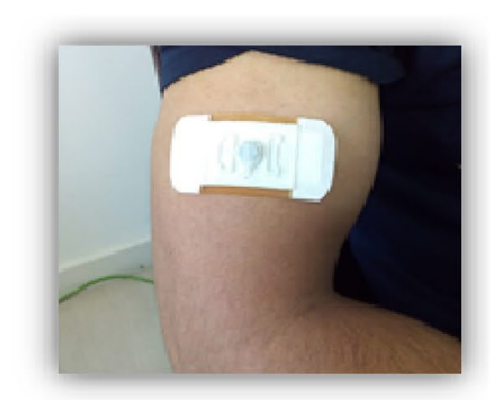

(b)

Fig. 1 a Conceptual view of device loaded with drugs b Device Prototype 


\subsection{Prototype development}

\subsubsection{Design of 3D model}

A rectangular shape was selected for the device model. The model was programmed using a 3D solid modeling software (Solidworks2014, Dassault Systems S.A) and saved in a stereolithography (.stl) file. The model was then imported into the 3D printer's software, and .stl format was converted to G-code for printing Fig. 2.

\subsubsection{D printing process}

ABS material was used for 3D printing. According to the CAD designs, device consisted of three parts:

- A $43 \times 30 \mathrm{~mm}$ platform

- A $6 \times 5.5 \mathrm{~mm}$ haptic motor housing compartment

- Two $17 \times 6 \mathrm{~mm}$ drug reservoirs

There were $11 \times 3 \mathrm{~mm}$ wide drug dispensation slits that were created at the ends of each reservoir for the drug delivery. The final prototype is shown in Fig. 3.

\subsubsection{Microcontroller of device}

The linear actuator used in this study is a vibration motor which can be driven by supplying a power of $3 \mathrm{~V}$ across the terminals. The goal of this study was to achieve the control over the vibrations; therefore, Arduino microcontroller was used. Microcontroller was programmed to switch on after $24 \mathrm{~h}$ to deliver drug each day Fig. 4

The Arduino was powered by $3 \mathrm{~V}$ regulated external power supply. After the initial testing, a specific voltage was selected and a microcontroller module was generated which stimulated the device for specific time interval with specific vibrational frequency.

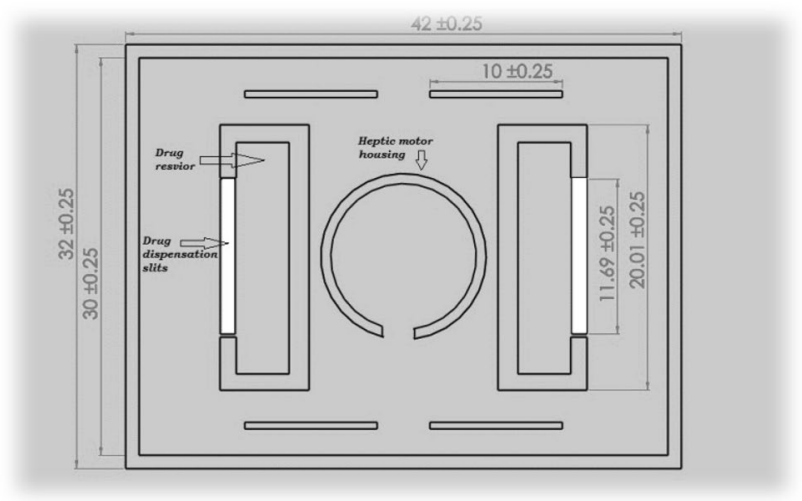

Fig. 2 CAD model of device

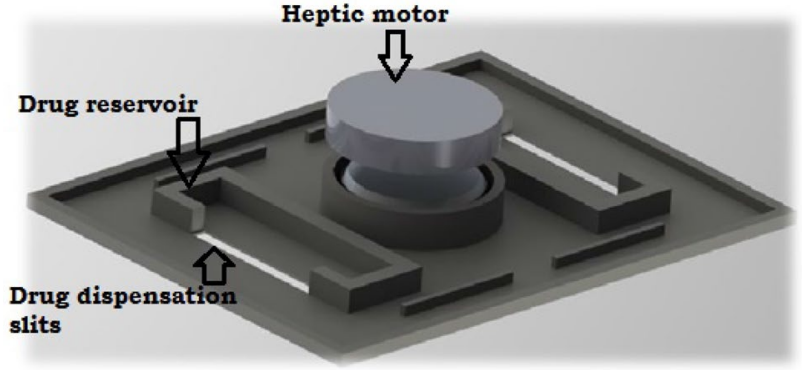

Fig. 3 3D printed device and its parts

\subsection{Drug release studies}

The pattern of drug release is greatly affected by many factors and constraints, i.e., vibrations frequency, voltage variations, time intervals and drug quantity. The release kinetics of device were analyzed by using diffusion method which is commonly used for transdermal drug delivery systems (TDDS). However, this was a novel study which could not utilize already reported testing beds for drug release studies; therefore, a specialized testing chamber was prepared for its drug release testing. This testing bed was a transparent closed chamber (shown in Fig. 5) which was hollow from inside and had a fixed place to hold dissolution beaker. Above the beaker, the device was mounted in a certain way that the drug fell precisely into the beaker.

This test comprised of three studies including in vitro dissolution testing, static study and accelerated wound conditions.

\subsubsection{In vitro dissolution testing}

The dissolution beaker containing $5 \mathrm{~mL}$ of PBS $(\mathrm{pH}=6.8)$ was fixed inside the chamber as described in Fig. 5. The device was allowed to release drug for a predetermined

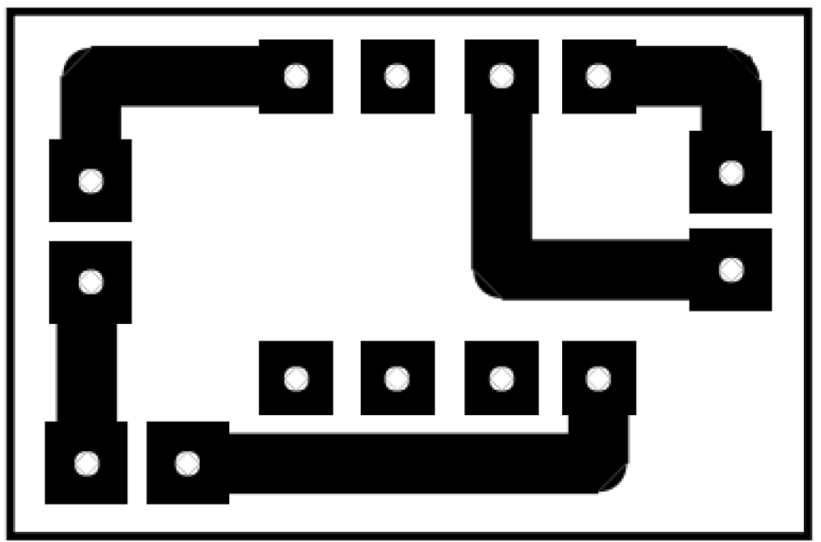

Fig. 4 Circuit diagram 
Fig. 5 Testing chamber

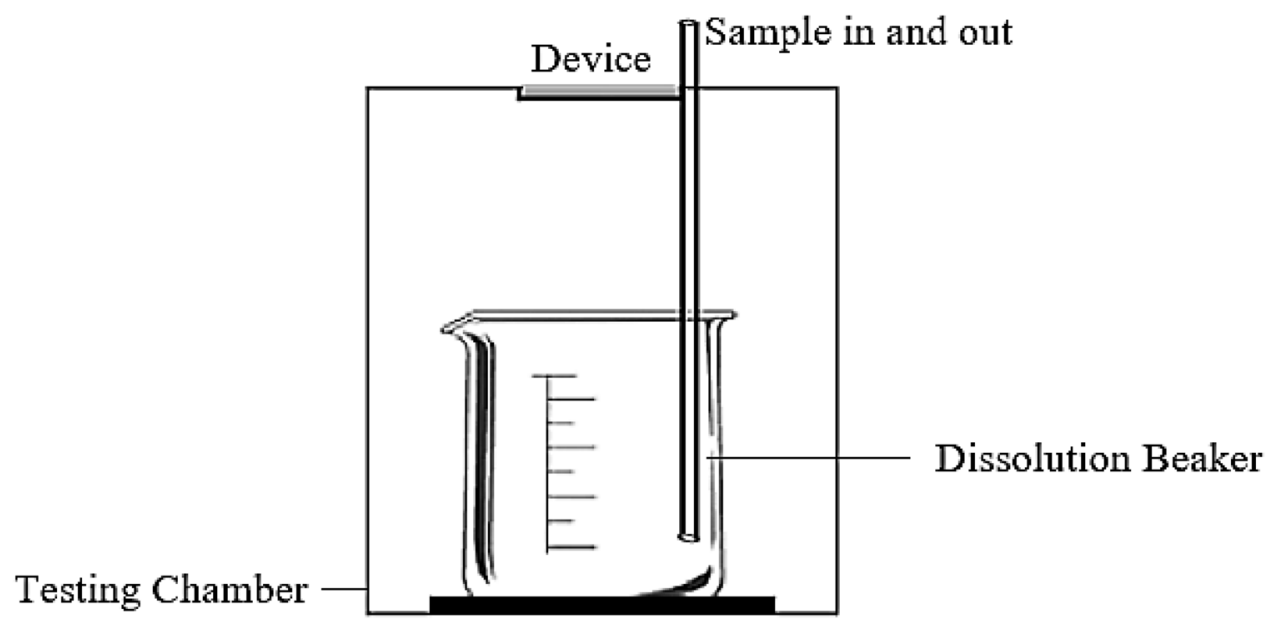

interval of time such as $300 \mathrm{~ms}, 500 \mathrm{~ms}, 800 \mathrm{~ms}, 1 \mathrm{~s}, 1.5 \mathrm{~s}$ and $2 \mathrm{~s}$. An aliquot of sample was withdrawn at specific time intervals, and the same amount of sample was replaced with fresh pre-warmed PBS. The absorbance of each sample was measured by UV-Vis spectrophotometry (JASCOV-530, JAPAN) at $390 \mathrm{~nm}$, and concentration was determined through the standard curve. All the studies were performed in triplicates.

\subsubsection{Drug release study (Static study)}

This device was designed to deliver drug for 5 days which is a recommended time period for topical drug delivery in case of acute wounds. Thus, for each time interval, drug was delivered for five consecutive iterations. This experiment was designed using Danckwert's theory which states, 'At the interface, the packet is able to absorb solute according to the laws of diffusion and is then replaced by a new packet of solvent.' Therefore, drug was delivered into the solvent only for one time, and then, the solvent was replaced by fresh solution for the next dosage, and this process continued until the last drug dosage. The absorbance of each sample was measured by UV-Vis spectrophotometry.

\subsubsection{Cumulative drug release study (Dynamic study)}

For this study, the same protocol as the static study was used; however, the only difference was that after the drug delivery in PBS, the whole $5 \mathrm{~mL}$ solution was not removed rather a little amount $(2 \mathrm{~mL})$ of drug was left there as residual drug. The fresh $3 \mathrm{~mL}$ solution was added in the beaker and second time drug was prepared. Afterward, UV spectrophotometry was performed on $3 \mathrm{~mL}$ solution to evaluate the concentration of drug.

\subsection{Microbial study}

In order to investigate the effectiveness of dosage regimen against bacteria, pure cultures of bacterial strains including Pseudomonas aeruginosa ( $P$. aeruginosa), Escherichia coli (E. coli), Methicillin-resistant Staphylococcus aureus (MRSA) were obtained from Mycovirus Research Lab, National University of Sciences and Technology (NUST), Islamabad.

In this study, well diffusion method was used to create zone of inhibition. The wells of same size were created on nutrient agar. Bacterial strains were grown on nutrient agar for $24 \mathrm{~h}$. The $100 \mu \mathrm{L}$ PBS solution containing different amounts of drug was loaded into the wells. The plates were incubated for $36 \mathrm{~h}$ at $37^{\circ} \mathrm{C}$. The area of zone of inhibition was measured by placing a ruler on the petri plate.

\subsection{Mechanical testing}

Due to cyclic load during manufacturing, packaging, transportation, clinician and patient handling and wearing, medical devices are expected to be strong enough to withstand all the pressure [8]. For that purpose, tensile, compression and bend test were carried out in universal testing machine following ASTM D638, ASTM D695 and ASTM D790 standards. These tests were performed using Ultimate tensile testing machine.

\subsection{Animal study}

BALB/c mice were purchased from National Institute of Health (NIH) Islamabad to check the feasibility of device and the effectiveness of the dosage regimen. The approval of ethical committee was obtained prior to the study 
(the approval letter has been attached at the end of the document).

The mice were divided into three groups and each group contained three mice. First group was negative control group on which no drug was applied, second group was positive control group on which a commercially available drug delivery system (Polyfax) was applied, and the third group was experimental group on which current drug delivery system was applied.

The wounds were created by following the protocol of incision wound model. The hair were shaved from the dorsal region of mice. After injecting the anesthesia, wounds of $10 \times 10 \mathrm{~mm}$ were created with the help of surgical blade by continuously scratching the skin.

Wounds of negative control group were covered with a simple gauze; wounds of the positive control group were treated with $0.1 \mathrm{~g}$ of Polyfax drug delivery system and the wounds of experimental group were covered with our device. The device was prepackaged to deliver for 5 days. After $24 \mathrm{~h}$ wounds, the qualitative and quantitative features were extracted and recorded. On second day, fresh dosage of polyfax was applied on positive group, as well as bandage was changed; however, in experiment group, only the electric stimulus was provided to deliver drug from the device. There was no need to change the bandage. The study continued for 5 consecutive days. Figure 6 demonstrates the procedure of in vivo study.

\subsubsection{Qualitative analysis of wound}

The observations were made to measure and grade the clinical parameters of the wound including wound color, humidity level, margins and surface, swelling and scar. The wounds were graded for each parameter after the careful observation. The following chart was followed for grading each parameter.

\subsubsection{Quantitative analysis of wound}

After the wound creation and device application, the diameter of wounds was measured to find out the wound closure rate. The area of wound was quantified with the help of a ruler. The results were recorded and demonstrated in the form of graph [11].

\section{Results and discussion}

\subsection{Release mechanism}

The proposed device is developed for controlled delivery of powdered drugs, and its release mechanism depends upon vibration movements of linear actuator. The mechanism is shown in Fig. 7. When the actuator vibrates upon receiving electrical stimulus, it transfers its vibrations to

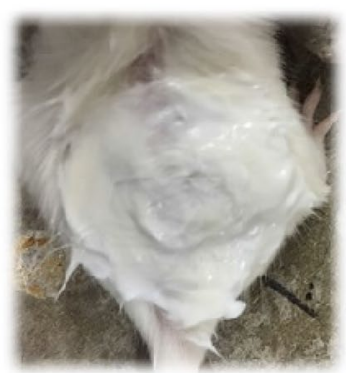

Application of hair removal cream

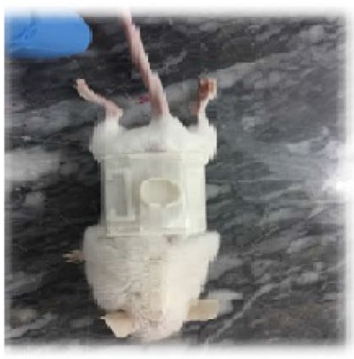

Device fixation

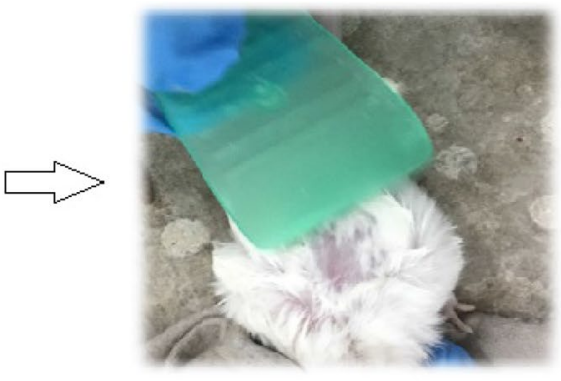

Hair removal
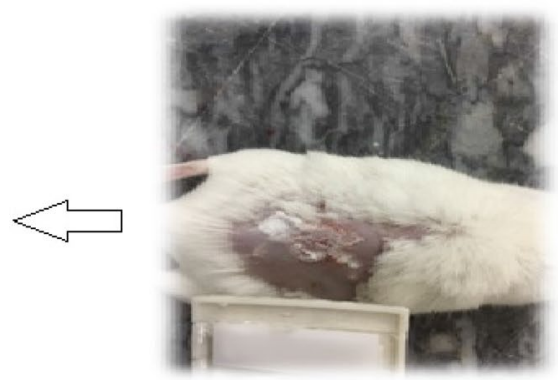

Drug application

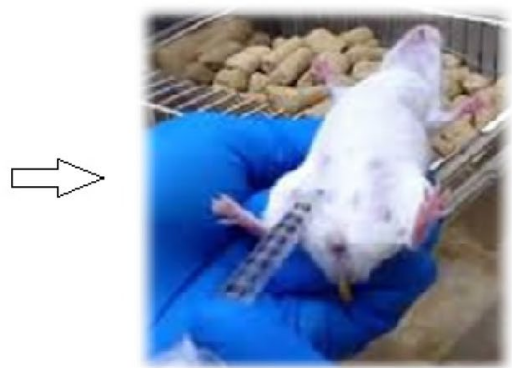

Anesthesia

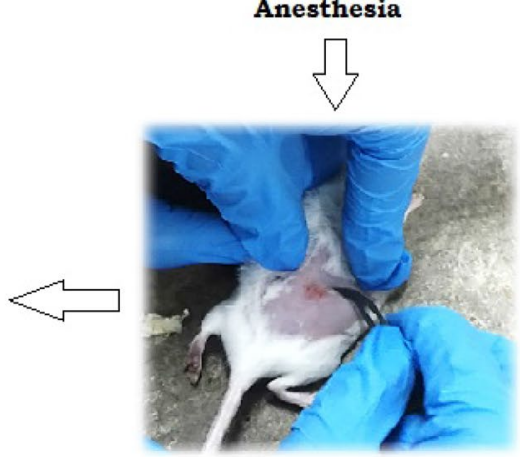

Wound Creation

Fig. 6 Wound creation steps 


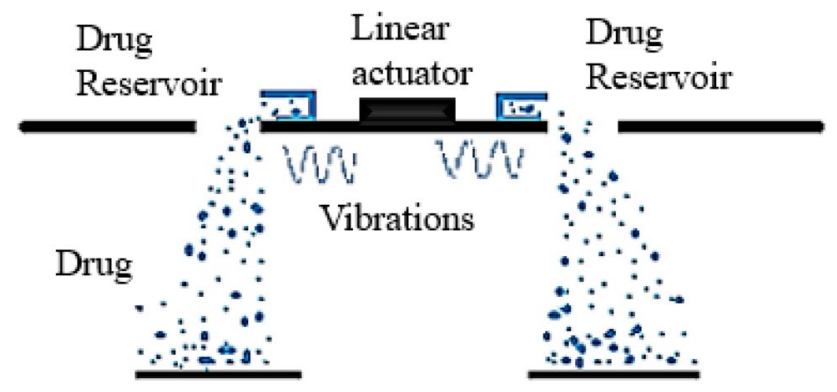

Fig. 7 Mechanism of drug release from the device upon actuator vibration (side view)

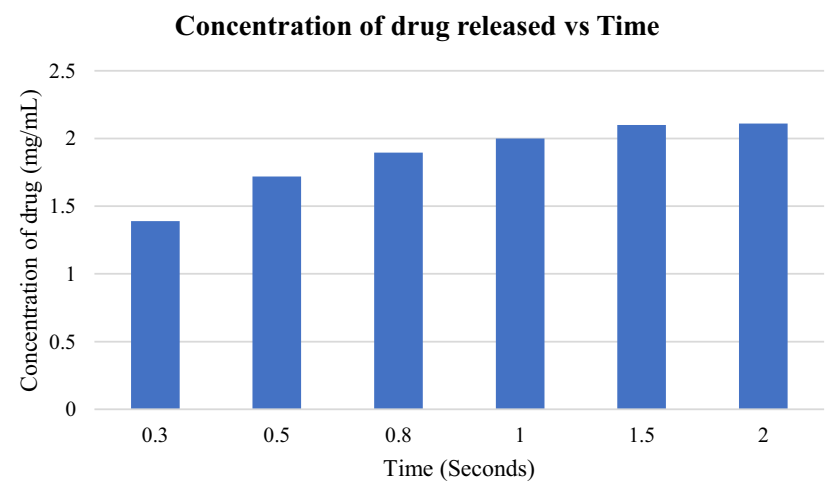

Fig. 8 In vitro drug release kinetics

the whole device. The drug inside the reservoir receives these vibrations and starts moving/vibrating inside the reservoir and as a result fells down from the slits. The device gives an immediate response to the electric stimulus which controls the drug release according to the patient requirements.

\subsection{Drug release studies}

The drug release patterns of the device were drawn through this study. A compliance chart of different dosage regimes with respect to time was built which a patient can use to determine his preferred dosing regimen.

\subsubsection{In vitro dissolution testing}

In vitro dissolution test was performed for six different time intervals of electrical stimulus which were $300 \mathrm{~ms}$, $500 \mathrm{~ms}, 800 \mathrm{~ms}, 1 \mathrm{~s}, 1.5 \mathrm{~s}$ and $2 \mathrm{~s}$. The UV absorption of each sample was taken on UV-Vis spectrophotometer, and percentage absorption was calculated with the help of standard curve. The concentration $(\mathrm{mg} / \mathrm{mL})$ of each time interval is given in Fig. 8.

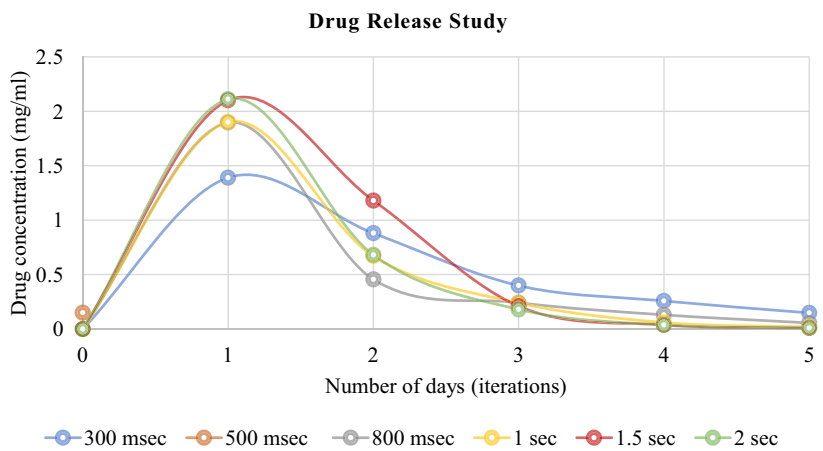

Fig. 9 Drug release pattern for five days

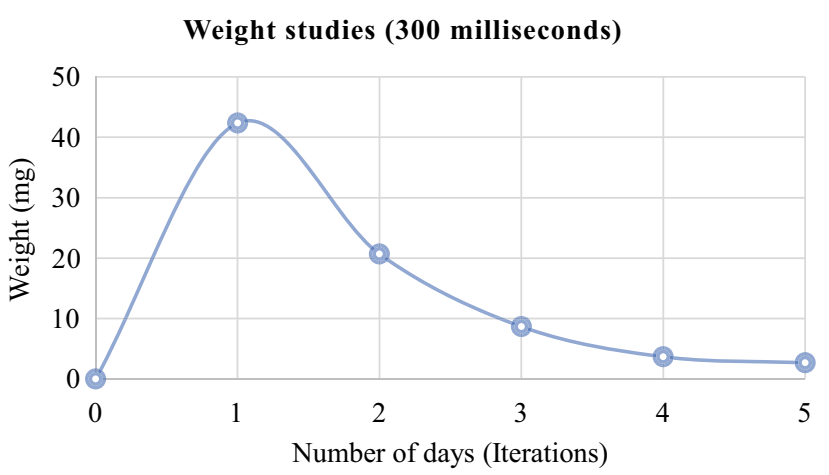

Fig. 10 Drug release profile with respect to weight

\subsubsection{Drug release study (Static study)}

The purpose of this study was to map out the drug delivery pattern for five consecutive days. The device was stimulated, and drug was delivered into fresh solution. The concentration of each solution was measured through UV-Vis spectrophotometer. The process was repeated for five iterations. The results of this study are given in Fig. 9.

The graph indicated that the best dosing regimen was at $300 \mathrm{~ms}$. At this time interval, device moderately delivered the drug from day 1 to day 5 .

The static study gave us the relationship between time interval and drug delivery in terms of concentration but most of the patients and clinicians talk in terms of weight of drug. So, the weight studies were performed to make the data more user friendly. Other purpose was to validate the static study results Fig. 10.

\subsubsection{Cumulative drug release study (Dynamic study)}

This study was performed to measure the release of the active pharmaceutical from a drug product matrix in a simulated laboratory environment. Figure 11 shows the results of in vitro studies, and it proved that device works 
Fig. 11 Cumulative drug release profile
Cumulative Drug Release Study

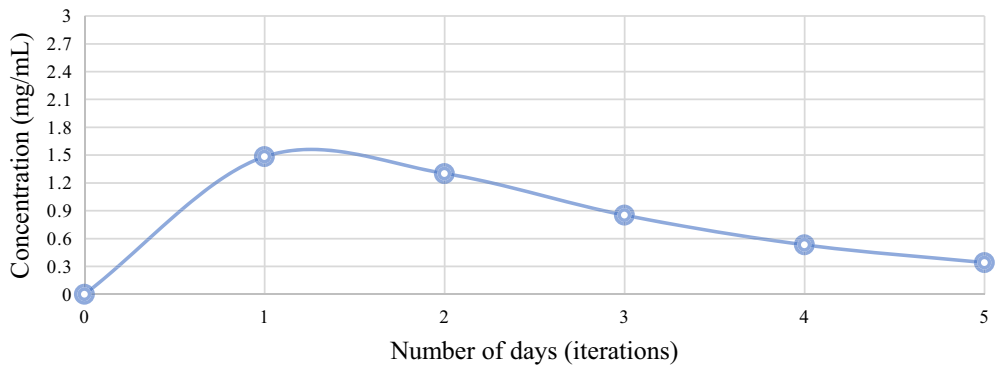

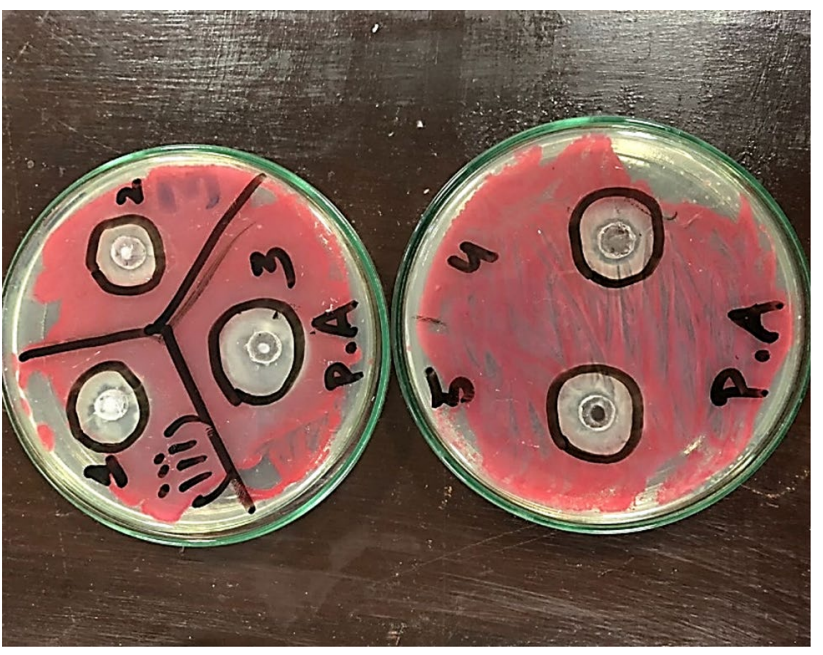

Fig. 12 Zone of inhibition of $P$. aeruginosa

better in wound conditions as it releases drug at effective therapeutic level.

In physiological conditions, the effect of drug dosage increases because of presence of residual drug on the wound site which gives a cumulative effect.

\subsection{Microbial study}

The purpose of this study was to evaluate the effect of dosage regimen on the microbes which cause delayed wound healing. The results of microbial study showed that drug is effective enough to create zones of inhibition around $1 \mathrm{~cm}$. The results of $E$. coli, MRSA and $P$. aeruginosa are given in the following figures. Zone of inhibition of each microorganism is given in Figs. 12,13 and 14. These experiments were performed in triplicates.

\subsection{Mechanical Testing}

In this study, tensile, compression and bend test were carried out and their results are given in Figs. 15, 16, 17 and 18. Tensile test was carried on both horizontal and vertical axes of device as the device is not a perfect

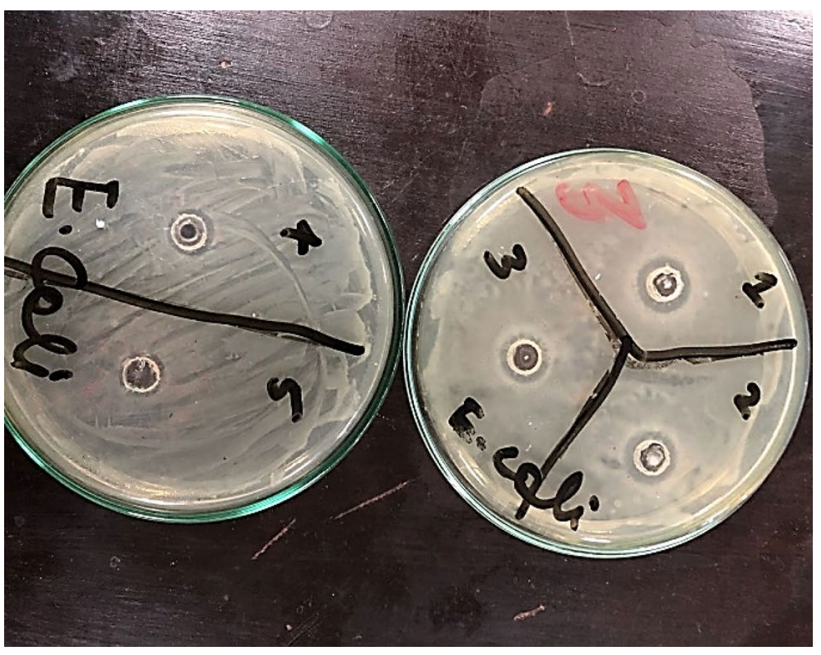

Fig. 13 Zone of inhibition of E. coli

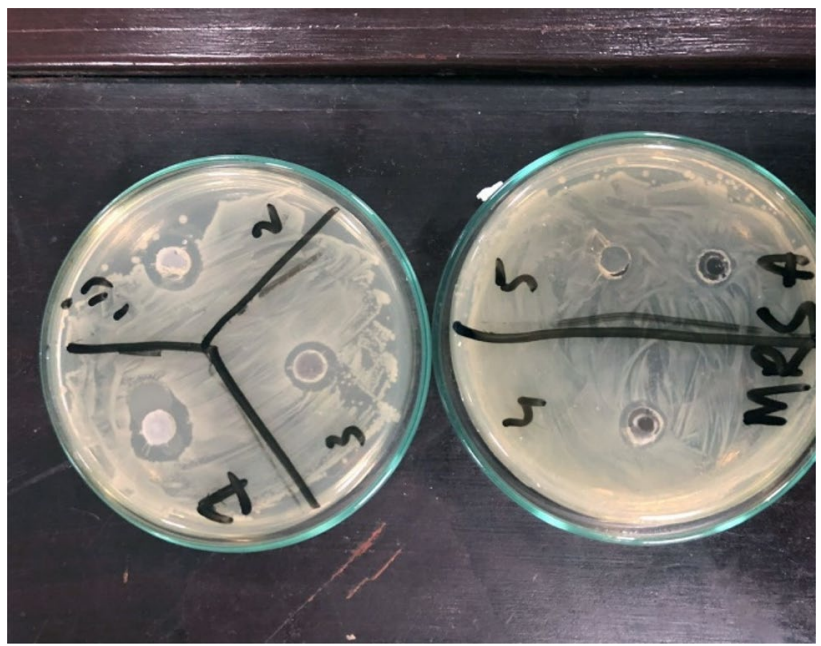

Fig. 14 Zone of inhibition of MRSA

square which makes the device behave differently on both axes and it is proven by the results shown in Figs. 14 and 15. The yield strength for horizontal and vertical axes is $1.93 \mathrm{MPa}$ and $2.69 \mathrm{MPa}$, respectively, and 


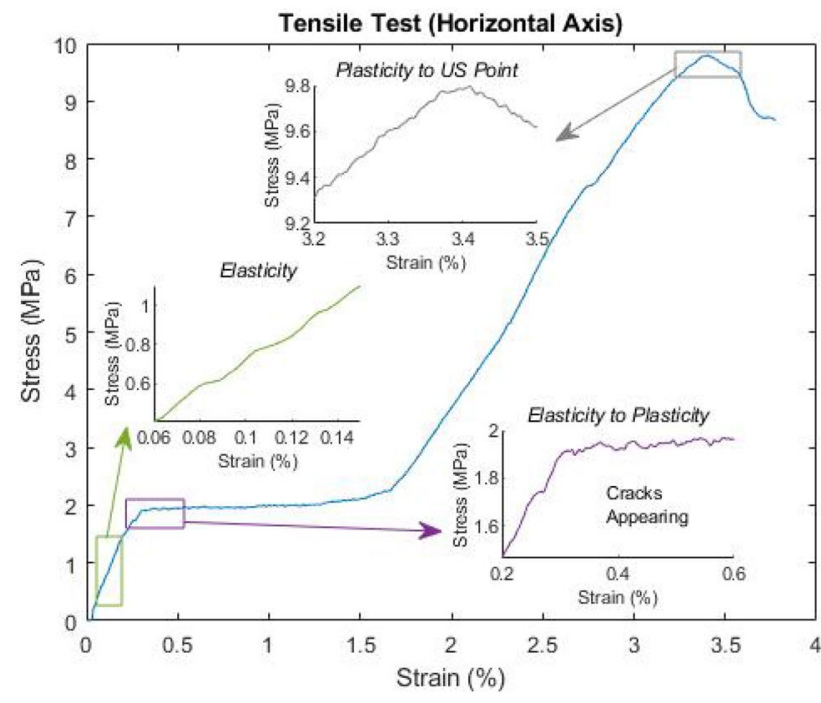

Fig. 15 Tensile strength of device in horizontal axis

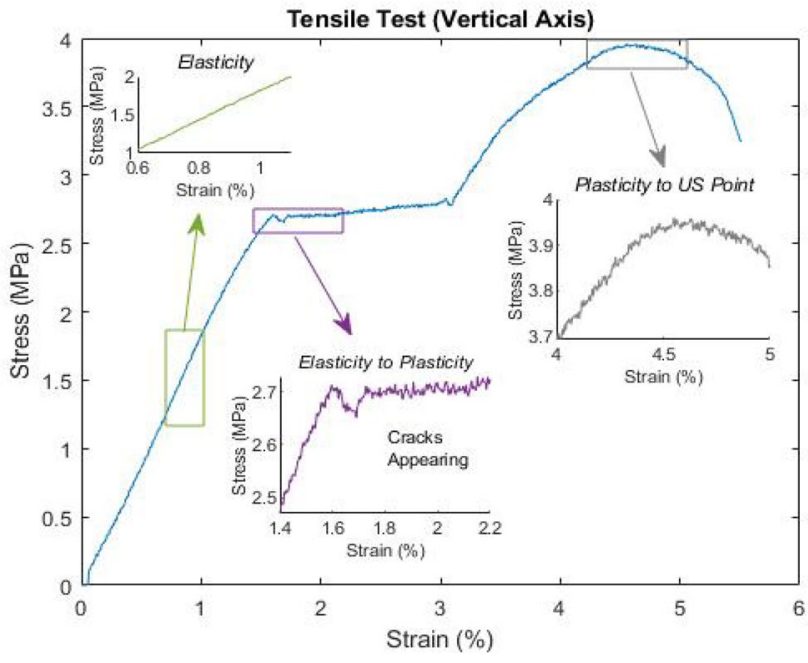

Fig. 16 Tensile strength of device in vertical axis

the ultimate tensile strength is $9.77 \mathrm{MPa}$ and 3.91. Figure 21 shows very small elastic region and a large plastic region which is the reason of high ultimate tensile strength value, but vertical axis went through a small plastic region.Fig. 16Tensile strength of device in vertical axisFig. 16Tensile strength of device in vertical axisFig. 16Tensile strength of device in vertical axis

Compression test was performed to check the durability of device under load, and Fig. 17 shows that device can endure a load of $43.87 \mathrm{MPa}$.

The bend test was performed to check the flexibility of device as the device can wrap around the body parts. Figure 18 shows the results of bend test which was carried out in ultimate testing machine.

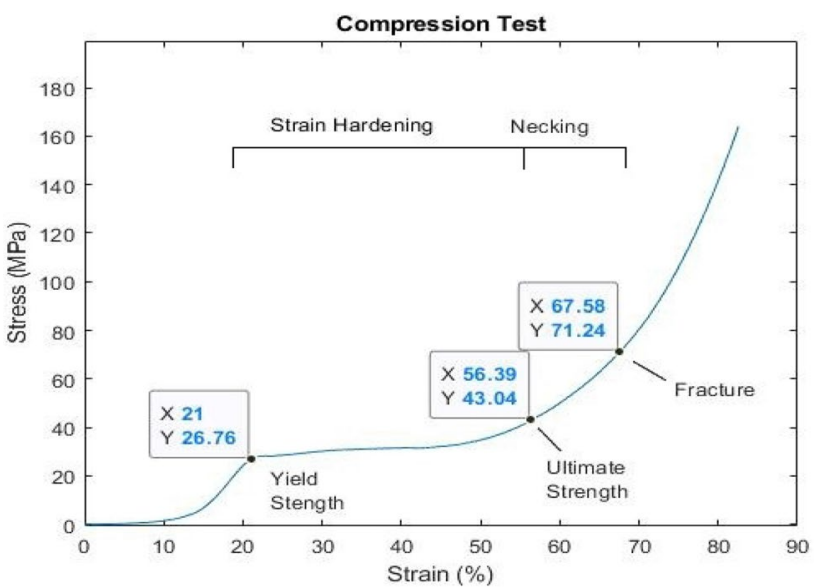

Fig. 17 Compression test of device

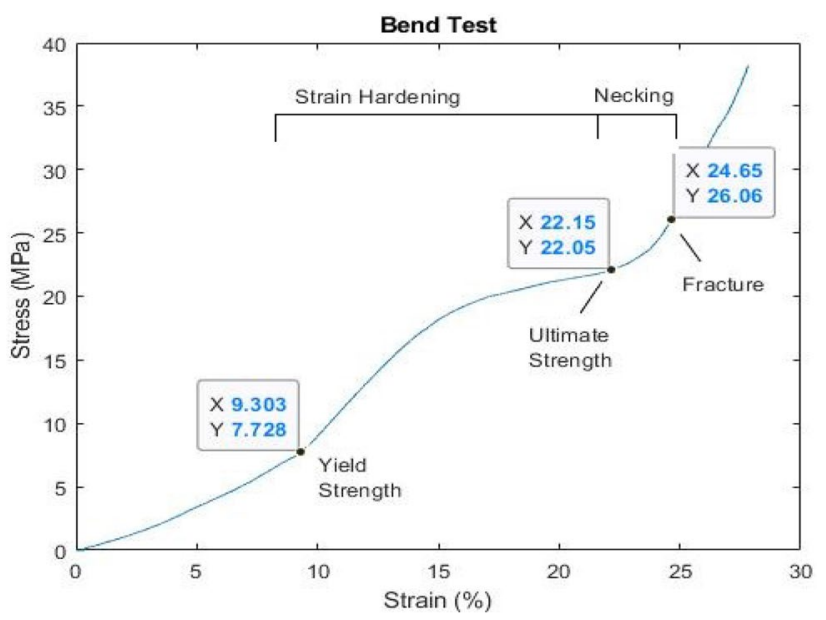

Fig. 18 Bend test of device

\subsection{Animal study}

Animal tests were performed on $B A L B / c$ mice, and the results were compared with the commercially available drug delivery system Polyfax.

\subsubsection{Qualitative analysis}

For qualitative analysis, the animals were divided into four groups, among them, two were experimental group and one was control group. The observations were made, and wounds are graded according to Table 1 . The results of qualitative analysis are given in Table 2 , and the wound healing is shown in Fig. 19.

The grading of wounds is given in Table 2 .

Due to study limitations, the histopathological assessment could not be performed; however, the observation of clinical parameters can give insights to the 
Table 1 Clinical parameters for qualitative analysis of wound healing $[9,10]$

\begin{tabular}{lll}
\hline Wound color & Red & 3 \\
\hline & Light red & 2 \\
& Brownish red & 1 \\
Humidity level & Wet & 0 \\
& Moist & 2 \\
Margins and Surface & Dry & 1 \\
& Rough, Irregular & 0 \\
& Rough, regular, Inflamed & 3 \\
& Smooth, even, regular & 2 \\
Swelling & Adheres to margins & 1 \\
& Hot, resistance to touch & 0 \\
& Red, painful movement & 3 \\
& Slight red, painful movement & 2 \\
Scar & No swelling & 0 \\
& Open wound & 3 \\
& Scab & 2 \\
& Closed wound & 1 \\
& Hairy wound & 0 \\
\hline
\end{tabular}

histopathological events of wound healing. During the study, the wound healing process did not show any clinical or gross sign of intercurrences including edema, erythema or suppuration. The absence of any abnormality during the healing process can be a potential indicator of absence of pathophysiological anomalies [12]. The figures were compared to the wound figures published by [12], and similarities were found in the healed founds as shown in Fig. 20.

The formation of primary fibrous scar was observed due to gradual maturation of the granulation tissues of the wound site. The fibrous scar formation is an important indicator of normal wound healing. After third day, the re-epithelialization of wounds was observed, and at 5th day, all three groups demonstrated full re-epithelialized wound surface. The re-epithelialized surface is achieved due to good keratinocyte proliferation and migration; therefore, we can claim the delivered dosage is good for normal keratinocyte proliferation and epithelialization.

\subsubsection{Quantitative analysis}

The diameter of wound was measured each day, and the results demonstrated that the rate of wound healing was similar for the first two days, and after the 3rd day, the healing rate increased exponentially for the wound healing device and the wound completely closed at the 5 th day; however, for the polyfax, the rate of healing was lesser than the wound healing device and it could close only 50 percent of wound even though it has more units (100 units) of drugs (polymyxin B sulfate and bacitracin zinc) as compared to Cicatrin (40 units) which contains neomycine and bacitracin zinc Fig. 21.

The reason could be in dose regime strategy as the polyfax releases the same amount of drug despite of the needs of wound and it also contains oils and petroleum minerals to ease the drug delivery. But the wound healing device releases drug according to the patient's demands; if patient needs more drug, it can release high concentration of drug and if the wound starts healing, it will release lesser amount of drug. So, this control over dosage regime showed accelerated healing and lesser usage of drug.
Table 2 Qualitative analysis of wound healing

\begin{tabular}{lllllll}
\hline Clinical Parameter & Mice \# & Day 1 & Day 2 & Day 3 & Day 4 & Day 5 \\
\hline Wound Color & 1 & 3 & 2 & 1 & 2 & 0 \\
& 2 & 3 & 2 & 1 & 1 & 1 \\
Humidity level & 3 & 3 & 2 & 1 & 1 & 0 \\
& 1 & 1 & 1 & 0 & 0 & 0 \\
Margins and surface & 2 & 1 & 1 & 0 & 0 & 0 \\
& 3 & 1 & 1 & 0 & 0 & 0 \\
\multirow{3}{*}{ Swelling } & 1 & 2 & 2 & 1 & 0 & 0 \\
& 2 & 2 & 2 & 1 & 1 & 0 \\
\multirow{3}{*}{ Scar } & 3 & 2 & 1 & 0 & 0 & 0 \\
& 1 & 3 & 2 & 1 & 0 & 0 \\
& 2 & 3 & 2 & 1 & 1 & 0 \\
& 3 & 3 & 2 & 1 & 0 & 0 \\
& 1 & 3 & 2 & 1 & 0 & 0 \\
& 2 & 3 & 2 & 1 & 1 & 0 \\
\hline
\end{tabular}



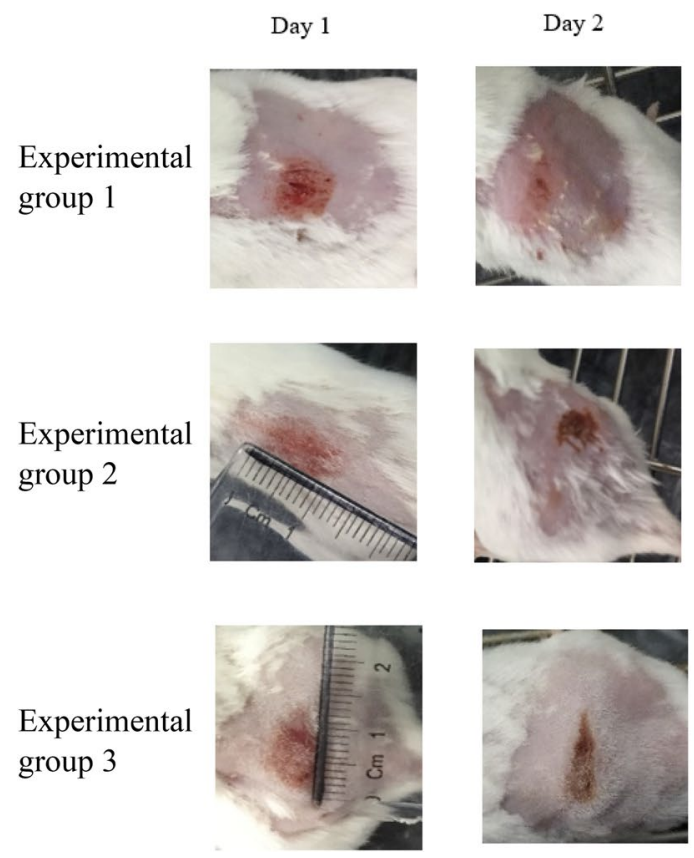

Experimental group 3

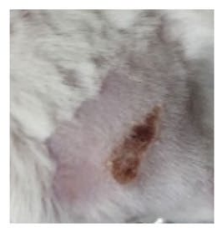

Day 4
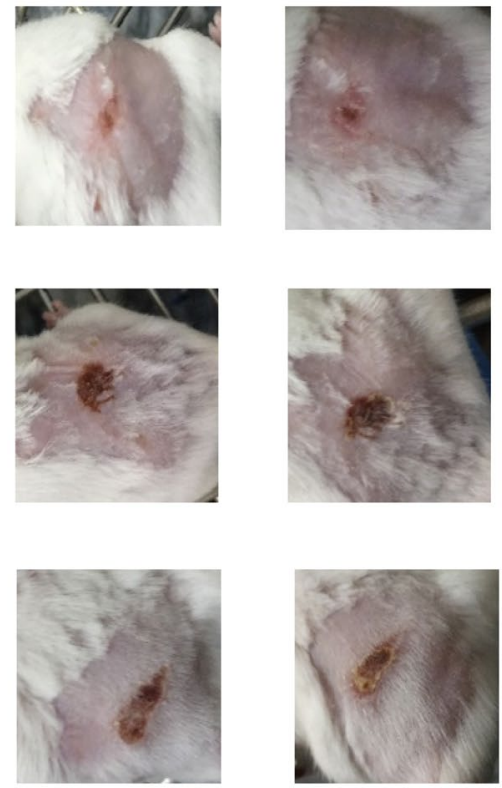

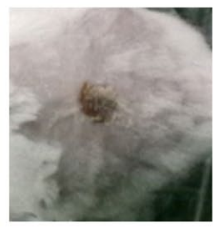

Day 5
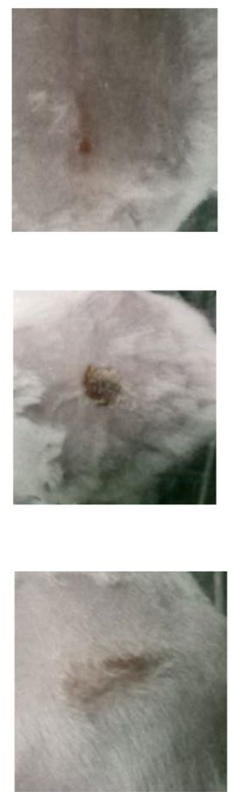

Fig. 19 Wound conditions of mice for 5 days

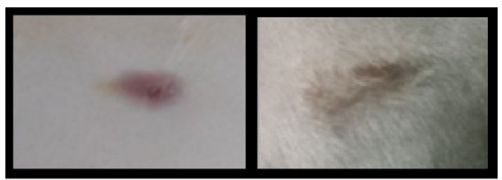

(a)

Fig. 20 a Healed wound from [12] b Healed wound of current study

\section{Discussion}

This study was aimed to develop and evaluate a novel drug delivery device for controlled and personalized drug delivery for wounds healing applications. The device was designed to inhabit the stimulating device and drug in the reservoirs. The stimulating device was a linear actuator which worked on the basis of haptic

technology. This technology uses kinaesthetic communication to provide stimulus; however, this stimulus is provided by either force or vibrations [13]. In this study, the linear actuator used vibrational energy to deliver the drug from reservoirs. The pattern of drug delivery was mapped out and calibration graph was made which helped in personalization of drug regimen for the patients.

The drug release from the reservoirs is dependent upon the frequency of the vibrations, timings of vibrations and the length of time intervals. The goal of this step was to map out the relationship between time interval and concentration of drug in a solution. As the drug was quantified in dissolved form, so it must follow some available models. To design the experiment, different dissolution theories were studied and Danckwerts theory was found fittest for our conditions. This theory states 'At the interface, the packet is able to absorb solute according to the laws of

Fig. 21 Wound healing rate

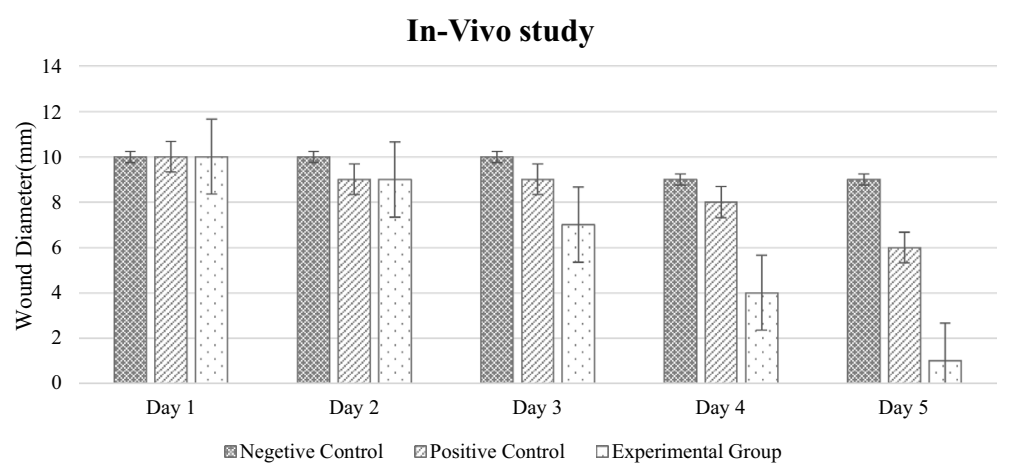


diffusion and is then replaced by a new packet of solvent.' Using this theory, the experiment was designed in such a way that the drug was delivered into the solvent only for one time, and for the next drug dose, the solvent was replaced by fresh solution and this process continued until the last drug dosage [14].

Dynamic conditions are the provision of simulated conditions in the laboratory by mimicking the actual wound conditions during drug delivery. In actual scenario, the wound is unable to utilize all of the drug that has been delivered on the first day of wound. As a result, when a specific amount of drug was delivered on second day, the wound not only contained the fresh second dose, but it would also contain the residual drug of previous day. As the drug dose being delivered from the device is different than the actual conditions, the modeling of actual conditions is a necessity to predict the behavior of device.

The role of bacteria in wound healing and wound deterioration has been debated for years but it has been established that some of the bacterial colonies are associated with the wound healing and some are associated with infections. All the skin wounds are colonized with bacteria, but they are only considered infected when the microbial load exceeds from $1 \times 10^{6} \mathrm{CFU}$ per gram of tissue mass [15]. So, the drug delivery systems try to be enough efficient to not to let the bacteria grow from certain range. Zones of inhibition of dosage regime of each day were measured to see if the device is releasing drug at effective therapeutic level or not. The well diffusion method was used to create zone of inhibition, and it was measured by placing a ruler on the petri plate. Three different strains, i.e., E. coli, MRSA and $P$. aeruginos $a$ were used as these are commonly present on the wounds as these bacteria are responsible for many hospital-borne infections and are commonly present on wound; if not controlled at early stages, it can cause infections and enter the wound into vicious non-healing cycle making them chronic wound.

To protect the patients from unforeseen events, medical devices are closely regulated and standardized according to the available international standards. There are well-defined and established standards for each aspect of device, and every device has to pass through it. ASTM standards are used to evaluate the basic properties, i.e., tensile strength, compression and bending ability. Due to application of load during manufacturing, packaging, transportation, clinician and patient handling and wearing, medical devices are expected to enough strong to withstand all of it. For that purpose, tensile, compression and bend test were carried out in universal testing machine following ASTM D638, ASTM D695 and ASTM D790 standards [15]. These tests are necessary to evaluate the component properties of a well-designed medical device.
For wound healing studies, different animal models, i.e., rodents, rats, rabbits, mice and guinea pigs are considered but still a gap remains between the animal and human wound healing. So, the animal models are chosen by keeping the scope of study and hypothesis of the test in mind. The scope of current study was to check the feasibility of device and the effectiveness of the dosage regimen, and this could be done on BALB/c mice as this is the simplest animal model with less anatomical complications and ethical concerns and also there are many similar studies which have been done on mice [16-19].

Incision wound model was selected for the current study, for wound creation because of the conditions, requirements and suitability of this study. The dose regimen of test device accelerated the wound healing rate exponentially after third day of study but for polyfax, the healing rate increased with a steady speed. Polyfax contains not only active ingredients Polymyxin B sulfate and Bacitracin zinc but also passive ingredients like oils and minerals to facilitate the drug penetration into skin. Consequently, lesser amount of active ingredients will be delivered; however, in the case of test device, cicatrin is delivered which only contains active ingredients. These ingredients help in faster healing of wounds.

The test device was developed and evaluated by many tests and found it effective for wound healing applications. The findings of this study can potentially open a new realm of wound care and replace existing wound care systems and significantly reduce burden of chronic wounds.

\section{Limitations}

This is study was carried out to develop a new concept in drug delivery systems; therefore, many aspects could not be studied. Another limitation was that the device was only studied in mice which are smaller animals. There is a need to study the device on larger animals before going for human studies.

\section{Conclusion}

Traditional drug delivery systems are failing to address the wound complications properly, leading them toward chronic realm. To avoid the burden of chronic wound, scientists are developing controlled release systems which can control the rate of drug according to the patient's needs. The current system provides a practical solution with an iterative design approach which provides drug according to the user's input and feedback. The effectiveness of the device is validated and proved through in vitro and pre-clinical studies. The current prototype system was 
developed with a priority of functions and features, and confirmation of feasibility of device. In addition to the wound care management functions provided by the current prototype system, new features and further technical improvements will be made for smarter and better wound care management in the future.

\section{Future prospects}

There are a few aspects which are planned to explore in future which includes addition of a strap to fix the device making it unable to move, studies on larger animals, i.e., rabbits or guinea pigs and histopathological studies of wounds.

\section{Acknowledgements No Acknowledgments}

Author contributions $\mathrm{SH}$ performed the experiments and wrote the paper. MNA supervised the whole study and critically reviewed the paper. MM helped to design the study and in manuscript drafting. $\mathrm{AA}$ and $\mathrm{MA}$ helped in experimentation.

Funding No funding was received for this manuscript.

Availability of data and material All the available data are given in the document.

\section{Declarations}

Conflict of interest On behalf of all authors, the corresponding author states that there is no conflict of interest.

Ethical approval Certificate is attached.

Code availability Not applicable.

Consent for publication All the authors are agreed to publish this manuscript.

Open Access This article is licensed under a Creative Commons Attribution 4.0 International License, which permits use, sharing, adaptation, distribution and reproduction in any medium or format, as long as you give appropriate credit to the original author(s) and the source, provide a link to the Creative Commons licence, and indicate if changes were made. The images or other third party material in this article are included in the article's Creative Commons licence, unless indicated otherwise in a credit line to the material. If material is not included in the article's Creative Commons licence and your intended use is not permitted by statutory regulation or exceeds the permitted use, you will need to obtain permission directly from the copyright holder. To view a copy of this licence, visit http://creativecommons. org/licenses/by/4.0/.

\section{References}

1. Dhivya S, Padma VV, Santhini E (2015) Wound dressings - a review. Biomedicine 5(4):22-22. https://doi.org/10.7603/ s40681-015-0022-9

2. Kirsner RS, Eaglstein WH (1993) The wound healing process. Dermatol Clin 11(4):629-640. https://doi.org/10.1016/S07338635(18)30216-X

3. Nwomeh BC, Yager DR, Cohen IK (1998) Physiology of the chronic wound. Clin Plast Surg 25(3):341-356

4. Sen CK (2019) Human wounds and its burden: an updated compendium of estimates. Adv Wound Care (New Rochelle) 8(2):39-48. https://doi.org/10.1089/wound.2019.0946

5. Patel VF, Liu F, Brown MB (2011) Advances in oral transmucosal drug delivery. J Control Release 153(2):106-116. https://doi.org/ 10.1016/j.jconrel.2011.01.027

6. Yui N, Kataoka K, Yamada A, Sakurai Y (1987) Novel design of microreservoir-dispersed matrices for drug delivery formulations: Regulative drug release from poly(ethylene oxide)- and poly(tetramethylene oxide)-based segmented polyurethanes. J Control Release 6(1):329-342. https://doi.org/10.1016/01683659(87)90086-1

7. Instruments T (2013) Haptics: Solutions for ERM and LRA Actuators. In.

8. Blaesi AH, Saka N (2016) Determination of the mechanical properties of solid and cellular polymeric dosage forms by diametral compression. Int J Pharm 509(1):444-453. https://doi.org/10. 1016/j.ijpharm.2016.05.020

9. Machmud E, Ruslin M, Waris R, Asse R, Qadafi A, Achmad H (2020) Effect of the application of chlorella vulgaris ointment to the number of fibroblast cells as an indicator of wound healing in the soft tissue of pig ears. Pesquisa Brasileira em Odontopediatria e Clínica Integrada. https://doi.org/10.1590/pboci.2020. 032

10. Vijaya KK, Nishteswar K (2012) Wound healing activity of honey: a pilot study. Ayu 33(3):374-377. https://doi.org/10.4103/09748520.108827

11. Xie Y, Gao K, Häkkinen L, Larjava HS (2009) Mice lacking $\beta 6$ integrin in skin show accelerated wound repair in dexamethasone impaired wound healing model. Wound Repair Regener 17(3):326-339. https://doi.org/10.1111/j.1524-475X.2009. 00480.x

12. Santos TS, Santos IDDD, Pereira-Filho RN, Gomes SVF, Lima-Verde IB, Marques MN, Albuquerque-Júnior RLCD (2021) Histological evidence of wound healing improvement in rats treated with oral administration of hydroalcoholic extract of vitis labrusca. Curr Issues Mol Biol 43(1):335-352

13. Sreelakshmi M, Subash TD (2017) Haptic technology: a comprehensive review on its applications and future prospects. Mater Today: Proceed 4(2):4182-4187. https://doi.org/10.1016/j.matpr. 2017.02.120

14. Horvath IR, Chatterjee SG (2018) A surface renewal model for unsteady-state mass transfer using the generalized Danckwerts age distribution function. R Soc Open Sci 5(5):172423-172423. https://doi.org/10.1098/rsos.172423

15. Bowler PG, Duerden BI, Armstrong DG (2001) Wound microbiology and associated approaches to wound management. Clin Microbiol Rev 14(2):244-269. https://doi.org/10.1128/CMR.14.2. 244-269.2001

16. Figueiredo Azevedo F, Santanna LP, Bóbbo VC, Libert EA, Araújo EP, Abdalla Saad M, Lima MHM (2017) Evaluating the effect of $3 \%$ papain gel application in cutaneous wound healing in mice. Wounds 29(4):96-101

17. Jeschke MG, Sadri AR, Belo C, Amini-Nik S (2017) A surgical device to study the efficacy of bioengineered skin substitutes 
in mice wound healing models. Tissue Eng Part $C$ Methods 23(4):237-242. https://doi.org/10.1089/ten.tec.2016.0545

18. Wosgrau ACC, Jeremias TDS, Leonardi DF, Pereima MJ, Di Giunta G, Trentin AG (2015) Comparative experimental study of wound healing in mice: Pelnac versus Integra. PLoS ONE 10(3):e0120322-e0120322. https://doi.org/10.1371/journal. pone.0120322

19. Yates C, Whaley D, Babu R, Zhang J, Krishna P, Beckman E, Wells A (2007) The effect of multifunctional polymer-based gels on wound healing in full thickness bacteria-contaminated mouse skin wound models. Biomaterials 28:3977-3986. https://doi.org/ 10.1016/j.biomaterials.2007.05.008

Publisher's Note Springer Nature remains neutral with regard to jurisdictional claims in published maps and institutional affiliations. 